\title{
Über die Entdeckung des Hibiscus storckii und seine Verwandtschaft zu Hibiscus rosa-sinensis
}

\author{
Hans-Georg Preissel
}

\begin{abstract}
The German gardener Berthold Seemann worked as a naturalist for the British Admiralty in the middle oft he 19th century. He explored the flora oft the Fiji islands and discovered the new species Hibiscus storckii. Today it is discussed whether Hibiscus storckii should be included in Hibiscus rosa-sinensis, which is a variable hybrid.
\end{abstract}

\section{Zusammenfassung}

Der deutsche Gärtner Berthold Seemann war Mitte des 19. Jahrhunderts im Auftrag der britischen Admiralität weltweit als Naturforscher tätig. Bei der Erfassung der Flora der Fidschi-Inseln entdeckte er Hibiscus storckii, eine neue Hibiscus-Art. Bis heute sind sich die Botaniker uneinig, ob es sich hierbei tatsächlich um eine eigene Spezies handelt oder ob diese Pflanze dem Formenkreis von Hibiscus rosa-sinensis zuzuordnen ist.

\section{Chancen in Kew Gardens für Berthold SEEMANN}

Es war der Beginn seiner internationalen Karriere als Botaniker, als der in Hannover geborene BERThold Seemann 1844 seine Heimatstadt im Alter von 19 Jahren verließ. Er ging nach London, um in den berühmten Königlich Botanischen Gärten von Kew seine gärtnerisch-botanischen Kenntnisse

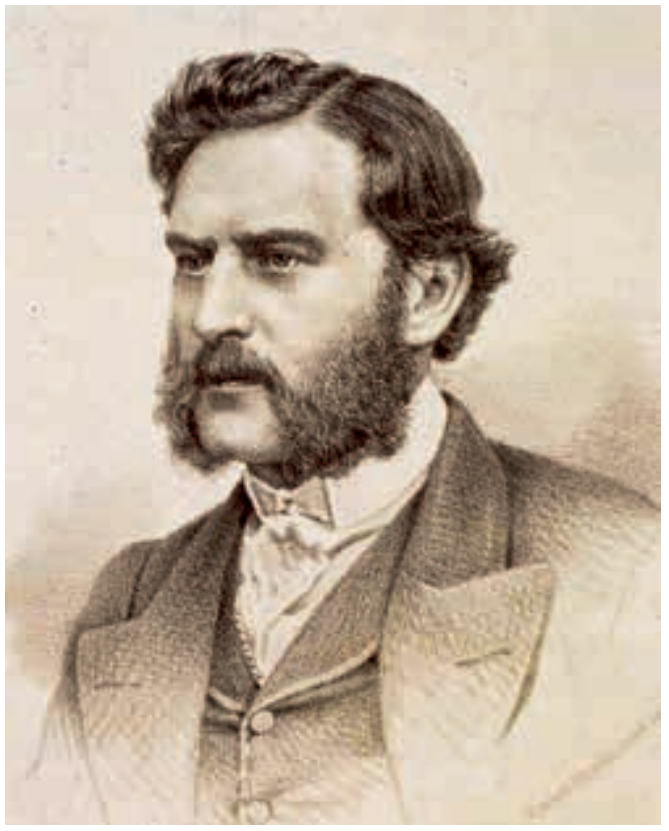

Abb. 1: Porträt von Berthold Seemann. (Foto: https:// upload.wikimedia.org/wikipedia/commons/9/94/Berthold_ Carl_Seemann.jpg) zu vertiefen. Seemann hatte damals gerade eine Gärtnerlehre in den Herrenhäuser Gärten beendet.

Der Wechsel eines Berufsanfängers im vorvorletzten Jahrhundert von Hannover nach London mag in der heutigen Zeit der so viel gepriesenen Freizügigkeit der Arbeitnehmerinnen und Arbeitnehmer innerhalb der Staaten der Europäischen Union erstaunen. Tatsächlich war es damals nicht ungewöhnlich, dass immer wieder Deutsche ihre Chancen im prosperierenden Großbritannien suchten. Dies beruhte durchaus auch auf den verwandtschaftlichen Beziehungen zwischen den Königshäusern von London und Hannover.

In den Gärten von Kew erhielten Berufsanfänger zweimal wöchentlich Unterricht und konnten sich dadurch u. a. in Geobotanik, Pflanzensystematik sowie Nutzpflanzenkunde weiterbilden. Dies geschah nicht allein zum Nutzen der jungen Gärtner. Schließlich waren die Königlichen Gärten Kew die Zentralstelle des Pflanzentausches für alle britischen Besitzungen im Ausland und den dort befindlichen botanischen Gärten. Dabei fiel dem Botanischen Garten in Kew auch die wichtige Aufgabe zu, die Nutzungsmöglichkeiten neu entdeckter Pflanzen zu untersuchen und deren Anbau in den Kolonien zu fördern. Und so ergab sich für den jungen deutschen Gärtner Berthold SeEMANN bereits im zweiten Jahr seiner Gehilfentätigkeit in London eine außergewöhnliche Chance. 


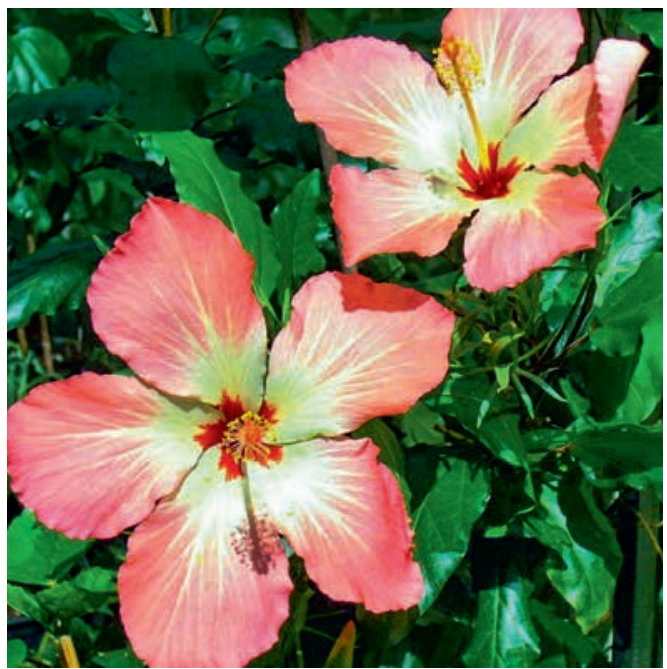

Abb. 2: Blüten von $H$. storckii (Foto: H.-G. Preissel)

\section{SEemann geht auf Weltumsegelung}

William J. Hooker, der Direktor der Gärten von Kew, empfahl nämlich den 21-jährigen hannoverschen Gärtner im Jahre 1846 der britischen Admiralität als Naturforscher für die Weltumseglung der Fregatte Herald. Die Fregatte hatte zwar schon ihre Fahrt begonnen, doch der zur Crew gehörende Naturforscher Thomas Edmonston war unerwartet ausgefallen. Er hatte bei einem Halt des Schiffes am 22. Januar 1846 in Ekuador durch das zufällige Entladen eines Gewehres sein Leben verloren. Berthold Seemann bekam den Auftrag, der Fregatte Herald nachzureisen. Am 17. Januar 1847 erreichte er Panama und konnte dort als neuer Naturforscher an Bord gehen. Es folgte eine 4 1/2jährige Weltumseglung von 1847 bis 1851 . Die dabei gewonnenen, umfangreichen naturwissenschaftlichen Erkenntnisse publizierte SEEMANN 1852 mit Unterstützung von Sir WILLIAM Hooker in dem Buch „The Botany of the Voyage of H. M. S. Herald“.

Man muss wohl mit der Arbeit des jungen SEEMANN sehr zufrieden gewesen sein, denn 1860 betraute ihn das britische Kolonial-Büro erneut mit einer Aufgabe. Sie betraf die in der Südsee gelegenen Fidschi-Inseln. Diese hatten 1859 angefragt, ob die britische Krone sie nicht in ihren Schutzbereich aufnehmen könne. Bevor man in Großbritannien eine Entscheidung über die offi- zielle Inbesitznahme treffen wollte, entsandte man eine Delegation unter der Leitung von Colonel Williams James Smythe, um Informationen über den möglichen Nutzen der Inseln einzuholen. Berthold Seemann fiel dabei die Aufgabe zu, eine Bestandsaufnahme sowohl der Wildpflanzen als auch der dort kultivierten Nutzpflanzen anzufertigen. Durch diese botanische Arbeit trug Seemann dazu bei, dass die Fidschi-Inseln 1874 Britische Kronkolonie wurden.

\section{Seemanns Arbeit an der Flora der Fidschi-Inseln}

Um die vor ihm liegende Arbeit auf den FidschiInseln besser bewältigen zu können, engagierte Seemann bei einem Zwischenaufenthalt in Sydney den jungen deutschen Jасов SтоRCK als Gehilfen. Dieser war als junger Gärtner nach Australien ausgewandert und arbeitete im Botanischen Garten von Sydney.

Nachdem Seemann 1862 einen Bericht „Über die Ressourcen und die Gemüseproduktion auf den Fidji-Inseln" vor beiden Häusern des britischen Parlaments präsentiert hatte, veröffentlichte er von 1865-1873 eine umfassende Flora der Fidschi-Inseln (Flora Vitiensis), die bis heute als Standardwerk anerkannt ist. SeEmann nutzte dafür die Unterstützung von Kew Gardens mit den dort tätigen Botanikern.

Bei der Namensgebung neu entdeckter Pflanzen verewigte SeEmann mehrfach den Namen

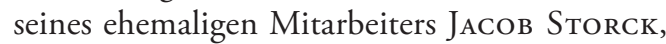
so bei Storckiella vitensis, einem Baum aus der Familie der Fabaceae. Sicherlich war dies eine An-

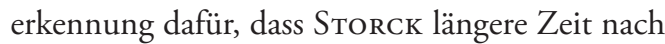
dem Ende der Expedition die Verbindung zu SEEMANN aufrechterhielt und Pflanzenproben von den Fidschi-Inseln nach Großbritannien sandte.

Aus Sicht der Pflanzenliebhaberei war Seemanns Entdeckung einer neuen HibiscusArt auf der Insel Taveuni nahe dem Ort Somosomo am interessantesten. SeEmann gab dieser auf den Fidschi-Inseln unter dem volkstümlichen Namen „Sequelu“ bekannten Pflanze den botanischen Namen Hibiscus storckii. Er beschrieb 
sie als einen sehr „unordentlich“ im Unterholz wachsenden Strauch, der sich von Hibiscus rosasinensis durch die stärker in die Länge gezogenen (länglich elliptischen) Blätter und die besonders schönen rosafarbenen Blüten auszeichnet. Eine so intensive rosa Farbe hatte Seemann bei H. rosasinensis noch niemals gesehen. Dennoch war er sich nicht völlig sicher, ob es sich bei seiner Entdeckung wirklich um eine neue Hibiscus-Art handelte, da die Pflanze ansonsten $H$. rosa-sinensis sehr ähnlich war.

\section{Hibiscus storckii und $\boldsymbol{H}$. rosa-sinensis}

Die Frage, ob es sich bei Hibiscus storckii um eine eigenständige Art handelt oder ob diese Pflanze dem Formenkreis von Hibiscus rosa-sinensis (Chinesischer Roseneibisch) zuzuordnen ist, wird bis heute von Botanikern unterschiedlich bewertet. Der Grund dafür ist im Wesentlichen in dem Prozess der wissenschaftlichen Weiterentwicklung bei der Erfassung der Vielfalt der Pflanzen und ihrer Benennung zu suchen.
Die zur Familie der Malvengewächse (Malvaceae) gehörende Gattung Hibiscus mit der Art H. rosa-sinensis wurde bereits 1753 von CARL von Linné in der Anfangszeit der von Linné begründeten und international anerkannten Pflanzenbenennung aufgestellt. Damals setzte sich die binäre Nomenklatur durch, die nur noch aus zwei Wörtern bestehenden wissenschaftlichen Pflanzennamen. Die jeden Pflanzennamen ergänzenden diagnostischen Artbeschreibungen waren hierbei auf 12 Worte begrenzt. So kommt es, dass den von Linné als Autor publizierten Arten zunächst nur wenige Informationen beigefügt waren, die über die Unterscheidungsmerkmale zu verwandten Arten informierten.

Bis heute hat sich die Botanik durch neue Untersuchungsmethoden und den damit entdeckten neuen Merkmalen an Pflanzen erheblich weiterentwickelt. Dies führt zwangsläufig auch zu einer Erweiterung und Veränderung der Regeln bei der Pflanzenbenennung. Parallel dazu hat der

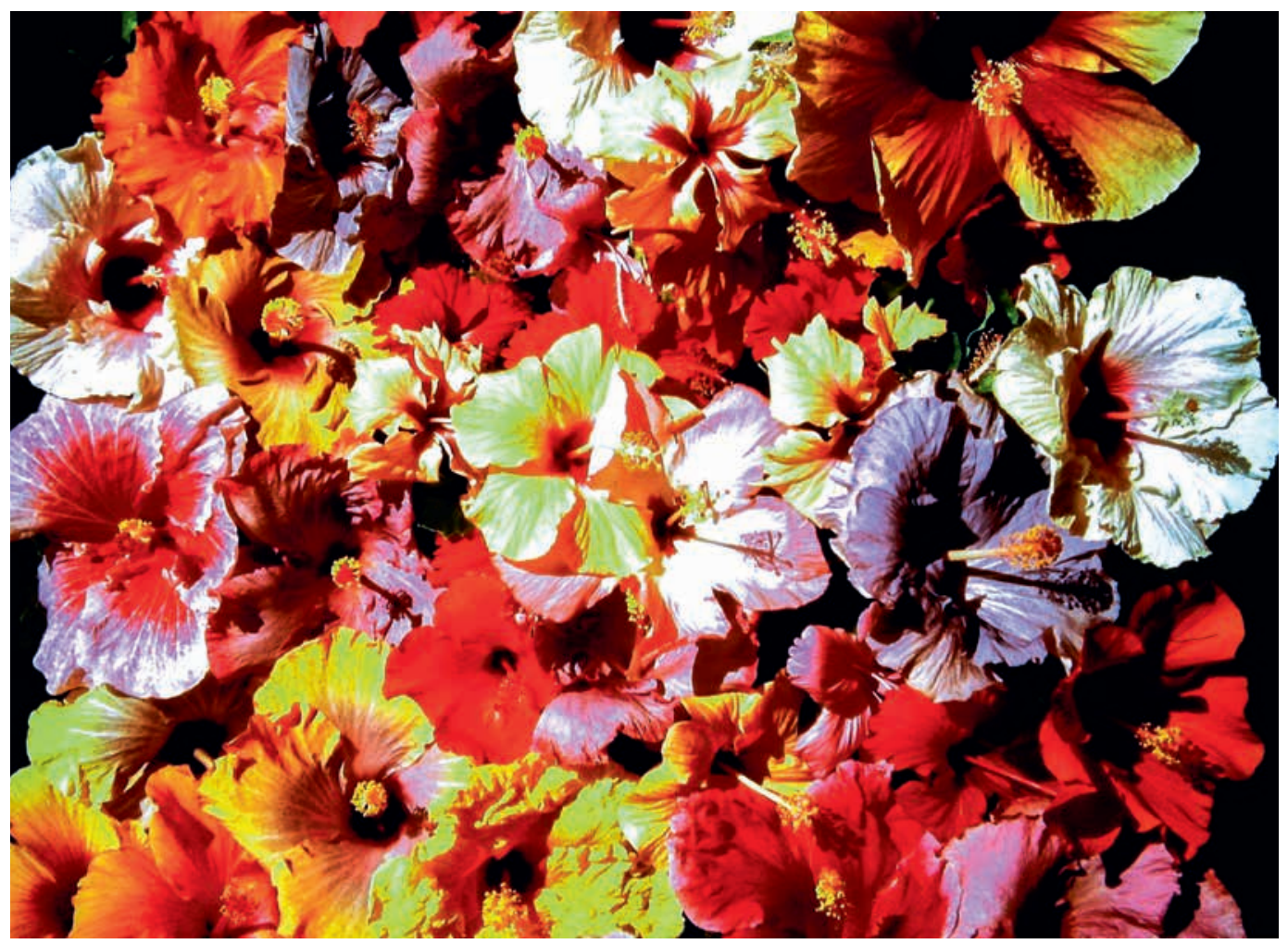

Abb. 3: Farbengemisch von Hibiscus-Blüten. (Foto: H.-G. Preissel) 
Mensch durch Kreuzungen von Nahrungs- und Zierpflanzen die durch die Natur gesteuerte Evolution der Pflanzen erheblich beeinflusst. Die sich infolge genetischer Durchmischung verschiedener Arten ergebende Neuerschaffung von Pflanzengruppen ist eine uralte Tradition.

Der Chinesische Roseneibisch (H. rosa-sinensis) gibt dafür ein eindrucksvolles Beispiel. Der unter diesem Namen von Linné beschriebene Pflanzentypus ist im Gewirr der genetischen Vermischung mit anderen Arten der Gattung Hibiscus sehr bald verloren gegangen, so dass die ursprüngliche Beschreibung der Art H. rosa-sinensis im Hinblick auf

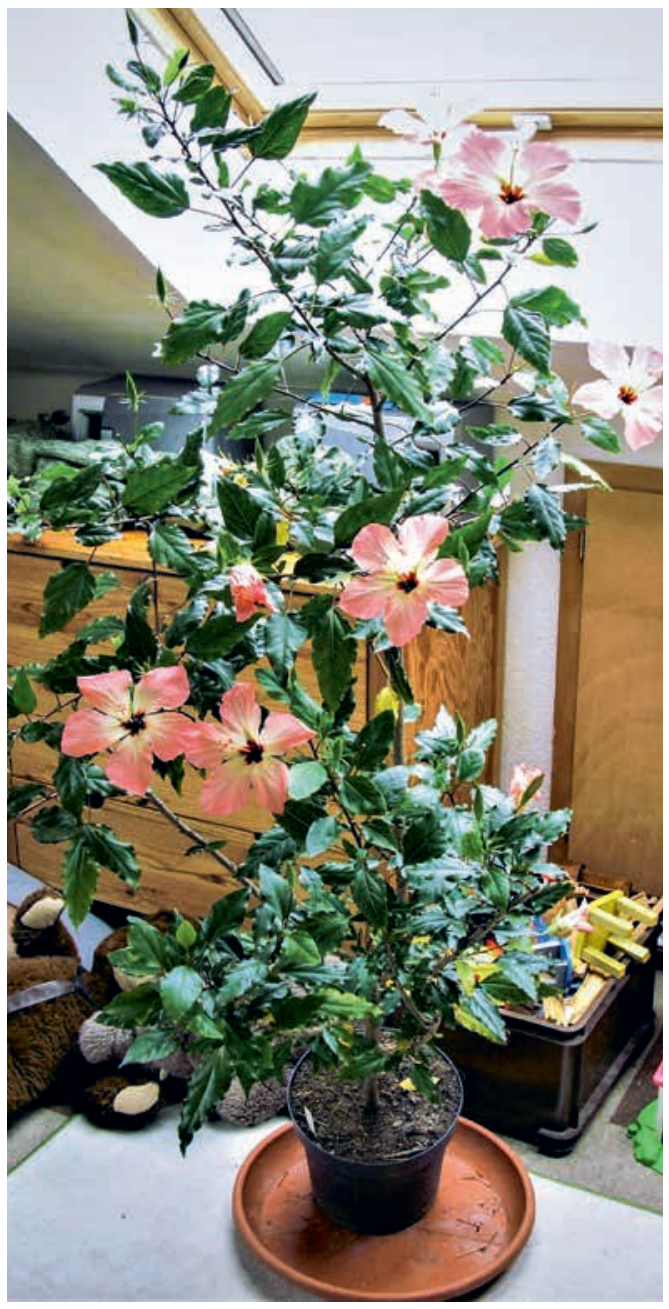

Abb. 4: Buschige Wuchsform von H. storckii.

(Foto: H.-G. Preissel) deren pflanzensystematische Stellung nicht mehr ergänzt werden konnte.

Aufgrund seiner attraktiven Blüten war der Chinesische Roseneibisch in den Tropen Asiens der wohl beliebteste Blütenstrauch. Bereits 1731 wurden erste Exemplare dieser Pflanze auch nach England eingeführt. Die Faszination, die von dieser Pflanze ausgeht, zeigt sich auch heute noch daran, dass ihre Blüten ein geläufiges Motiv auf Hawaii-Hemden sind und dass Frauen auf Hawaii und den Pazifischen Inseln ihre Haare mit Hibiscus-Blüten schmücken. Wird eine Blüte über dem rechten Ohr getragen, bedeutet dies, dass die Frau nicht gebunden ist. Hat sie eine feste Bindung, schmückt sie ihr linkes Ohr.

Aufgrund der enormen Schmuckwirkung des Hibiscus ging es vielen Pflanzensammlern bereits in der Vergangenheit nicht so sehr um eine botanische Erfassung der verschiedenen Hibiscus-Arten, sondern um das Experimentieren und Züchten neuer Blütenfarben und -formen. So erlebte der Chinesische Roseneibisch um 1810 in China mit einer großen Vielfalt an Blütenfarben bereits einen Höhepunkt in der züchterischen Entwicklung. Als seine Heimat wurden damals die tropischen Gebiete in China und Indien vermutet. Doch ist seine genaue botanische Heimat, d. h. ein Gebiet, wo diese Pflanzenart natürlich vorkam und durch kontinuierliche Fortpflanzung ihre genetischen Eigenheiten bewahren konnte, bis heute unbekannt. Auffällig ist, dass sich offenbar viele alte Fundorte von $H$. rosa-sinensis an Stellen befanden, die an der Seeroute von China nach Indien lagen und regelmäßig von Handelsschiffen angelaufen wurden. Neben Handelsgütern wie Tee- und Gewürzpflanzen wurden von diesen Schiffen auch viele Zierpflanzen mitgenommen und dadurch weltweit verbreitet.

Die kurze Beschreibung der von Linné erfassten Spezies H. rosa-sinensis einerseits und das Bestreben möglichst viele ähnliche Pflanzen mit den unterschiedlichsten Blütenfarben zu kreuzen andererseits führte dazu, dass die heute unter diesem Namen kultivierten Zierpflanzen den Rahmen der ursprünglich beschriebenen Wildart sprengen. 
Dem botanischen Namen Hibiscus rosa-sinensis wurde deshalb heute in dem Internationalen Code der Nomenklatur der Kulturpflanzen eine Sonderstellung eingeräumt: Er bezeichnet eine genetisch komplexe Pflanzengruppe, nämlich ein Hybridgemisch aus etwa acht verschiedenen $\mathrm{Hi}$ biscus-Arten, die alle untereinander kreuzbar sind. Aus diesen Kreuzungen hervorgegangene Auslesen können sortenecht nur vegetativ vermehrt werden. Zwecks Wiedererkennung werden diese gärtnerisch kultivierten Auslesen in aller Regel durch einen Sortennamen ergänzt. Dadurch wird auch der Eindruck vermieden, dass es sich bei den Pflanzen um Abkömmlinge der reinen Wildart handelt.

In der botanischen Nomenklatur werden Kreuzungen zwischen verschiedenen Arten - also Hybriden - normalerweise durch ein zwischen Gattungs- und Artbeinamen eingefügtes „x“ gekennzeichnet. Dies würde den Namen Hibiscus x rosa-sinensis ergeben. Dem multiplen Artgemisch

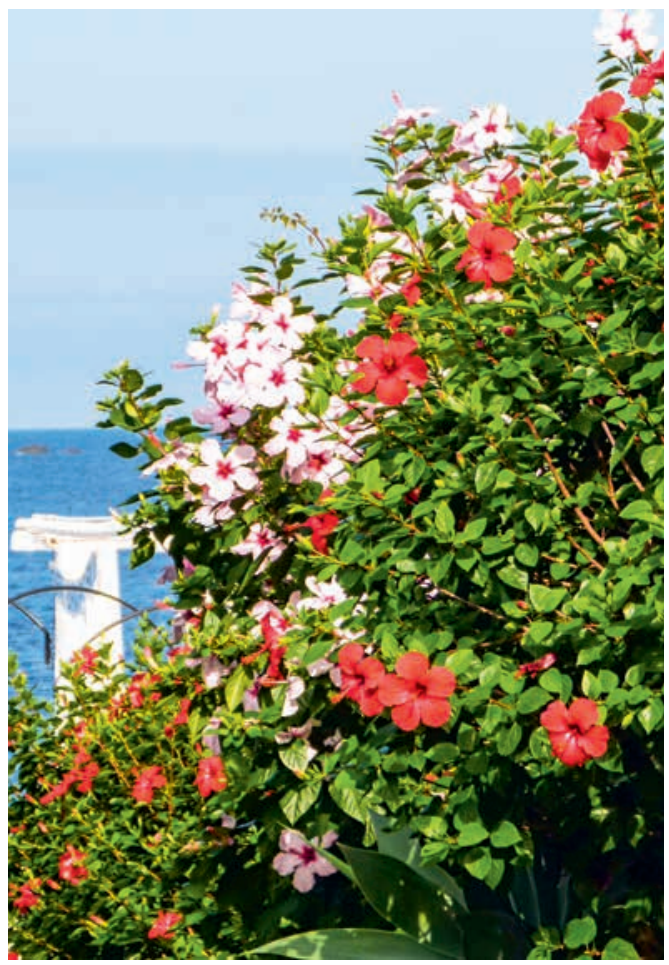

Abb. 5: Roseneibisch erfreut sich überall großer Beliebthei. Hier üppige Exemplare auf Panarea, Liparische Inseln. (Foto: H. Steinecke) von Hibiscus rosa-sinensis wird jedoch eine Sonderstellung eingeräumt, weil man bei ihm auf solch eine formelhafte Bezeichnung verzichtet.

Von manchen Botanikern wird auch Hibiscus storckii nicht als eigenständige Art angesehen, sondern dem Hybrid-Gemisch von Hibiscus rosa-sinensis zugeordnet, da sie mit allen Sorten des Chinesischen Roseneibischs kreuzbar ist. Für die Einstufung von $H$. storckii als eigenständige Art spricht jedoch, dass diese nachweislich nur auf den Fidschi-Inseln zu den in der Natur vorkommenden Pflanzen gehörte und dass die Nachkommen aus Samen vermehrter H. storckii in ihren Merkmalen sehr einheitlich sind. Dies trifft sowohl auf Morphologie und Farbe der Blüten als auch auf die länglichen Blätter zu. Die Blüten bleiben mit bis zu $12 \mathrm{~cm}$ Durchmesser eher klein. Die einzelnen Blütenblätter sind jeweils etwa $6 \mathrm{~cm}$ lang und $4 \mathrm{~cm}$ breit. Auf ihrer gesamten Länge überlappen sich die Blütenblätter nicht, so dass

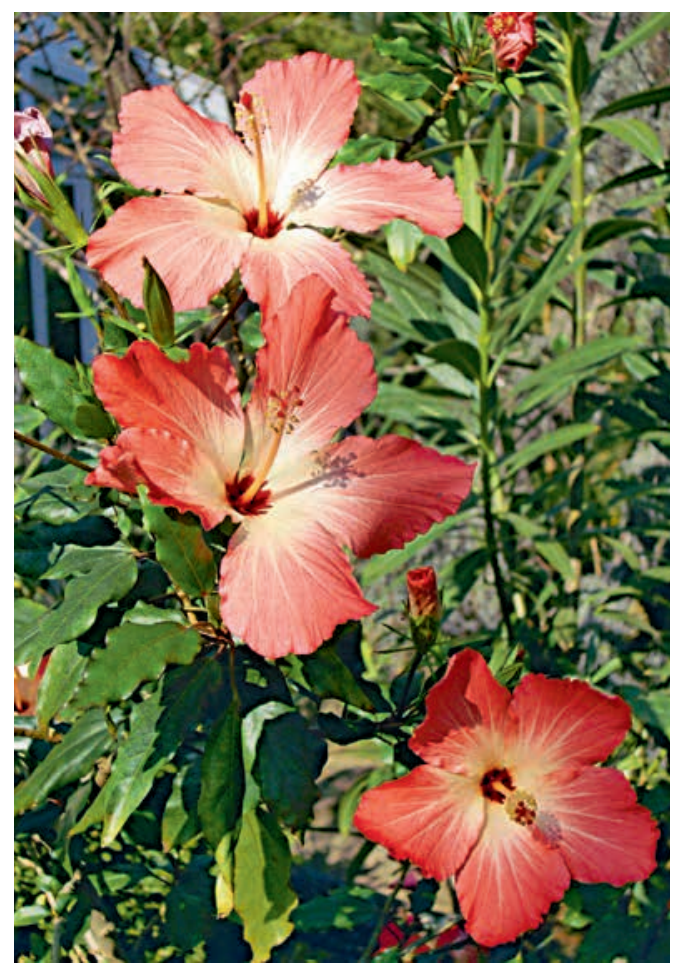

Abb. 6: Hibiscus storckii sollte wegen seiner schönen großen Blüten häufiger als Zierpflanze verwendet werden. (Foto: H.-G. Preissel) 
H. storckii eine offene Blütenform aufweist, die auch als Windmühlentyp bezeichnet wird. Bei der Züchtung der Hybriden von Hibiscus rosa-sinensis ist dagegen eine weitgehend geschlossene Blütenform vorherrschend, so dass sich bei den neueren Sorten die Blütenblätter in der Regel über die gesamte Länge überlappen.

Im Zuge des Wachstums neigen Pflanzen von $H$. storckii zur Ausbildung vieler Seitentriebe, wobei der Mitteltrieb relativ schwach bleibt. Dadurch entstehen reich verzweigte Büsche, die in der Regel nur eine maximale Höhe von 2-3 m erreichen und damit kleiner bleiben als Sämlinge von Hibiscus rosa-sinensis.

\section{Suche nach Hibiscus-Urformen}

Auf der Suche nach Hibiscus-Urformen reiste Ross H. Gast, einer der Gründer der American Hibiscus Society, 1963 auf die Fidschi-Inseln. Dort hoffte er auch Pflanzen des damals in den botanischen Sammlungen äußerst seltenen H. storckii zu finden. Er nahm Kontakt zu dem Arboretum und auch dem Botanischen Garten in der Hauptstadt Suva auf und musste erfahren, dass den Fachleuten keine Naturstandorte von $H$. storckii mehr bekannt waren. Dennoch suchte Ross GAsT intensiv weiter, denn er hoffte, die gesuchte Hibiscus-Art noch in Hausgärten von Kokosnuss-Pflanzern oder in Sammlungen von Hibiscus-Liebhabern zu finden. Doch Ross Gast fand nicht die von den Fidschi-Inseln stammende, rosa-blühende Hibiscus-Art, dafür aber in großer Zahl die aus Hawaii stammenden Hibiscus-Züchtungen mit ihren großen, farbenprächtigen Blüten, die auch im Süden der Vereinigten Staaten ihren Siegeszug angetreten hatten.

Obwohl Storcks Roseneibisch aus dem warmen Klima der Südseeinseln stammt, ist er auch bei uns eine attraktive, leicht zu pflegende, ganzjährig blühende Zierpflanze. Diese kann als Zimmer- oder Kübelpflanze gehalten werden. In der Freilandsaison der Kübelpflanzen von Mai bis September steht sie am besten an einem möglichst sonnigen Platz. Dort verträgt sie problemlos auch kühle Nachttemperaturen um $10{ }^{\circ} \mathrm{C}$, wenn tagsüber das Thermometer auf mindestens $18{ }^{\circ} \mathrm{C}$ ansteigt. Während der Überwinterung im Haus oder Wintergarten sollten die Temperaturen bei mindestens $16^{\circ} \mathrm{C}$ gehalten werden.

\section{Literatur}

Anonymus 1996: Ponderings. The Seed pod. - Amer. Hib. Soc. Oct. 12/1996.: 20.

Bates, D. M. 1965: Notes on the cultivated Malvaceae, 1. Hibiscus. - Baileya 13: 56-96.

Beers, L. \& Howie, J. 1995: Growing Hibiscus. - Nashville, Tennessee.

BRETSCHNEIDER, E. 1898: History of european botanical discoveries. - Press of the Imperial Russian Academy of Sciences.

Davis, P. H. \& Heywood, V. H. 1973: Principles of angiosperm taxonomie. - New York.

Gast, Ross H. 1980: Hibiscus around the world. American Hibiscus Society, Cocoa Beach, Florida.

KNoLL, J. 2016/17: Berthold Seemann - Vom Gärtnergehilfen in Herrenhausen zum weltreisenden Entdecker. - Naturhistorica Hannover 158/159: 55-79.

Phillips, R. H. 1990: Hibiscus storckii fact or fiction. Second International Australian Hibiscus Soc. Inc. Convention.

Preissel, U. U. H.-G. 2008: Der Chinesische Roseneibisch als Kübelpflanze. - Gartenpraxis 2008/2: 49-59.

RÜMPLER, T. 1882: Illustriertes Gartenbau-Lexikon. - Hamburg.

Seemann, B. 1853: The botany of the voyage of H. M. S. Herald. Auszug in Hamburger Garten- und Blumenzeitung 1853, in mehreren Fortsetzungen.

Seemann, B. 1865-73: Flora Vitiensis. - London.

Suringar Valckenier 1927: Die Anwendung der internationalen botanischen Nomenklaturregeln. - Mitteilungen d. Deutschen Dendr. Ges. 1927: 293-333.

Wettstein, R. 1935: Handbuch der Systematischen Botanik. - Leipzig.

\section{Anschrift des Autors:}

Dr. Hans-Georg Preissel, Meisenweg 2, 30826 Garbsen, E-Mail: georg.preissel@web.de 